Ann. Zootech., I979, 28 (4), 38I-392.

\title{
Influence de la saison sur le comportement alimentaire des moutons recevant des fourrages verts en zone tropicale humide
}

\author{
Brigitte MICHALE'T-DOREAU (1) et A. XANDÉ \\ avec la collaboration technique de F. NIPAU
}

Station de Recherches zootechniques

Centre de Recherches A gronomiques des Antilles et de la Guyane, I.N.R.A., 97 r7o Petit Bourg (Guadeloupe)

\begin{abstract}
Résumé
On a étudié les variations saisonnières des quantités de matière sèche ingérées ainsi que les modifications du comportement alimentaire et mérycique des moutons recevant, au cours des deux saisons sèche et humide, des repousses de Brachiaria decumbens et de Digitaria swazilandensis âgées de 35 ou 56 jours.

Les quantités ingérées sont significativement plus faibles au cours de la saison humide :

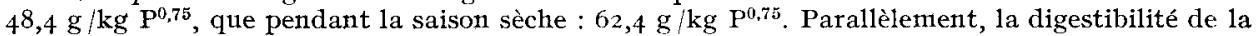
matière sèche passe de $59,8 \mathrm{p}$. I oo en saison humide à 56,8 p. Ioo en saison sèche et la teneur en matières azotées des repousses de 35 jours est plus faible au cours de la saison humide : 12,2 p. Ioo contre I 5,5 en saison sèche. D'autre part, 1" "indice de température effective " ou indice de confort thermique calculé en fonction de la température et de l'humidité de l'air est en moyenne plus élevé au cours de la saison humide $\left(+\mathrm{I}, 3^{\circ} \mathrm{C}\right)$, l'augmentation atteignant $+3^{\circ} \mathrm{C}$ au cours de la période de mesure des deux repousses âgées de 35 jours.

Les variations saisonnières de durée unitaire d'ingestion, de rumination et de mastication passent respectivement de $7,37 \mathrm{mn} / \mathrm{g} . \mathrm{kg} \mathrm{P} \mathrm{P}^{0,75}$ en saison humide à 6,02 en saison sèche, de $\mathrm{I} 0,80$ à 8,46 et de I8, I 9 à I 4,44 et le nombre de repas passe de 6,7 à 8,4 .

Les variations saisonnières des quantités ingérées sont difficiles à expliquer. Elles semblent dépendre à la fois des modifications des conditions climatiques (variations de l'indice de température effective, bien que celui-ci ne représente peut-être pas au mieux l'état de confort thermique de l'animal), qui entraîneraient une modification de la capacité d'ingestion des animaux et des variations de digestibilité et de teneur en matières azotées, donc des variations d'ingestibilité du fourrage lui-même.
\end{abstract}

(I) Adresse actuelle : Laboratoire des Aliments, Centre de Recherches de Clermont-Ferrand, I.N.R.A., C.R.Z.V. de Theix, 63 I ro Beaumont (France). 


\section{Introduction}

La digestibilité et la quantité d'aliments ingérée sont les deux principaux facteurs qui déterminent la quantité d'éléments nutritifs consommée par un ruminant disposant de sa ration à volonté. Leur importance relative est variable suivant la nature ou l'origine du fourrage, mais dans le cas des fourrages verts tropicaux, le facteur prépondérant est la quantité de matière sèche volontairement ingérée par l'animal (MILFORD et MrNson, 1965). En effet, pour des fourrages de digestibilité comparable, la quantité ingérée peut varier dans des limites très importantes, de 35 à $80 \mathrm{~g} / \mathrm{kg} \mathrm{p}^{0,75}$ (MILFORD et Minson, I965). En distribuant des repousses d'âge constant à des bovins ou à des caprins, BUTTERWORTH, GROOM et WILSON (I96I) et ChENosT (I972) ont respectivement montré que les quantités de fourrage volontairement ingérées sont plus faibles pendant la saison humide que pendant la saison sèche. Cette diminution d'ingestion, pour des fourrages de même âge, peut être attribuée soit à une modification de l'ingestibilité du fourrage lui-même, soit à tune variation de la capacité d'ingestion de 1'animal.

On sait que l'ingestibilité d'un fourrage dépend principalement de l'effet d'encombrement qu'il exerce dans le rumen, c'est-à-dire de la vitesse de dégradation de la masse des membranes, mais aussi de son appétibilité. On sait aussi que la capacité d'ingestion d'un animal varie avec ses besoins énergétiques et plus particulièrement ses besoins de production, mais qu'elle peut aussi être modifiée par l'état nutritionnel et sanitaire de l'animal ainsi que par de nombreux stress, stress thermique par exemple.

Pour essayer de préciser la part revenant à chacun de ces facteurs, ingestibilité du fourrage ou capacité d'ingestion de 1'animal, dans les variations saisonnières des quantités ingérées, nous avons enregistré la consommation par des moutons de 4 fourrages au cours de la saison sèche et de la saison humide, ainsi que le comportement alimentaire et mérycique de ces animaux.

\section{Matériel et méthodes}

\section{Conditions de milien}

Cet essai s'est déroulé à la Station de Recherches Zootechniques du C.R.A.A.G. en Guadeloupe, dont le climat tropical humide se caractérise par deux saisons, une saison sèche de février à juin et une saison pluvieuse de juillet à janvier. Les essais ont été réalisés respectivement de mars à mai et de septembre à novembre; la température et 1'humidité relative ont été enregistrées journellement et la pluviométrie moyenne au cours de la période de mesures a été relevée par la Station de Bioclimatologie du C.R.A.A.G.; les conditions climatiques moyennes lors de chaque période de mesures ont été rapportées dans le tableau I et leur variation moyenne au cours d'une journée, sur la figure $\mathrm{I}$.

A partir de ces données, nous avons calculé un indice de confort thermique 
TABLEAU

Conditions climatiques, composition Climatic conditions, chemical

\begin{tabular}{|c|c|c|c|c|c|c|c|}
\hline \multirow{2}{*}{$\begin{array}{c}\text { Fourrages } \\
\text { Species }\end{array}$} & \multirow{2}{*}{$\begin{array}{c}\text { Age } \\
\text { Age } \\
\text { (jours) } \\
\text { (days) }\end{array}$} & \multirow{2}{*}{$\begin{array}{l}\text { Saison } \\
\text { Season }\end{array}$} & \multirow{2}{*}{$\begin{array}{l}\text { Pluviométrie } \\
\text { Pluviometyy } \\
(\mathrm{mn} / \mathrm{j}) \\
(\mathrm{mn} / \mathrm{d})\end{array}$} & \multirow{2}{*}{$\begin{array}{l}\text { Température } \\
\text { moyenne } \\
\text { Mean } \\
\text { temperature } \\
\left({ }^{\circ} \mathrm{C}\right)\end{array}$} & \multicolumn{2}{|c|}{$\begin{array}{l}\text { Humidité relative } \\
\text { Relative humidity } \\
\text { (p. Ioo) }\end{array}$} & \multirow{2}{*}{$\begin{array}{l}\text { Température } \\
\text { effective } \\
\text { Effective } \\
\text { temperature } \\
\left({ }^{\circ} \mathrm{C}\right)\end{array}$} \\
\hline & & & & & Minimum & Maximum & \\
\hline \multirow[t]{2}{*}{ Brachiaria. } & 35 & $\begin{array}{l}\text { Sèche } \\
\text { Dry }\end{array}$ & 3,9 & 24,4 & $5^{8,0}$ & 85,0 & 22,0 \\
\hline & & $\begin{array}{l}\text { Humide } \\
\text { Wet }\end{array}$ & $x 6,4$ & 27,1 & 65,0 & $8 \mathrm{r}, 0$ & 25,5 \\
\hline \multirow[t]{2}{*}{ Brachiaria. } & $5^{6}$ & $\begin{array}{l}\text { Sèche } \\
\text { Dry }\end{array}$ & 4,9 & 25,4 & 62,5 & 86,0 & 22,9 \\
\hline & & $\begin{array}{l}\text { Humide } \\
\text { Wet }\end{array}$ & I 3,4 & 25,2 & 74,0 & 89,0 & 22,8 \\
\hline \multirow[t]{2}{*}{ Digitaria. . } & 35 & $\begin{array}{l}\text { Sèche } \\
\text { Dry }\end{array}$ & $2, \mathrm{I}$ & 24,0 & 57,0 & 90,0 & 22,0 \\
\hline & & $\begin{array}{l}\text { Humide } \\
\text { Dry }\end{array}$ & 12,4 & 26,7 & 69,0 & 86,0 & 24,5 \\
\hline \multirow[t]{2}{*}{ Digitaria } & $5^{6}$ & $\begin{array}{l}\text { Sèche } \\
\text { Dry }\end{array}$ & 4,5 & $26, \mathrm{I}$ & 63,6 & 88,0 & 23,9 \\
\hline & & $\begin{array}{l}\text { Humide } \\
\text { Wet }\end{array}$ & 3,2 & $25, \mathrm{r}$ & $7^{\mathrm{r}, \mathrm{O}}$ & 86,0 & 23,2 \\
\hline
\end{tabular}

Parallèlement nous avons enregistré le comportement alimentaire et mérycique des animaux (RUCKeBusch, I963) par transcription graphique, sur une bande enduite de noir de fumée, des variations de pression subies par un ballonnet rempli de mousse de polyuréthane et fixé sous la mâchoire de 1'animal par des sangles souples. La durée unitaire d'ingestion, de rumination ou de mastication correspond au temps nécessaire pour ingérer, ruminer ou mastiquer un gramme de matière sèche de fourrage $/ \mathrm{kg} \mathrm{P}^{0.75}$. Nous avons appelé " repas " une période d'ingestion d'une durée égale ou supérieure à $7 \mathrm{mn}$. Dès qu'une interruption à l'intérieur d'une période d'activité donnée excède $7 \mathrm{mn}$, deux périodes sont comptées.

Minson et Milford (I967) ayant montré sur des fourrages tropicaux que les quantités de matière sèche ingérées étaient en liaison étroite avec le pourcentage de matière sèche digérée après I2 heures d'incubation in vitro, nous avons mesuré la digestibilité in vitro I 2 heures pour chaque fourrage (méthode de TinLEY et TERRY, I963).

\section{Résultats}

\section{Composition chimique et digestibilité}

Nous avons rapporté, au tableau I, les variations saisonnières de la composition chimique des 4 fourrages utilisés dans cet essai. En moyenne, ces variations 
chimique et digestibilité des fourrages

composition and grass digestibility

\begin{tabular}{|c|c|c|c|c|c|c|c|c|c|}
\hline \multicolumn{4}{|c|}{$\begin{array}{l}\text { Composition morphologique } \\
\text { Morphological composition }\end{array}$} & \multicolumn{4}{|c|}{$\begin{array}{l}\text { Composition chimique (p. Ioo) } \\
\text { Chemical composition }\end{array}$} & \multicolumn{2}{|c|}{$\begin{array}{l}\text { Digestibilité } \\
\text { Digestibility }\end{array}$} \\
\hline $\begin{array}{c}\text { Feuilles } \\
\text { Leaf }\end{array}$ & $\begin{array}{l}\text { Tige } \\
\text { Stem }\end{array}$ & $\begin{array}{l}\text { Epis } \\
\text { Ear }\end{array}$ & $\begin{array}{l}\text { Débris } \\
\text { Re- } \\
\text { mains }\end{array}$ & $\begin{array}{l}\text { Matière } \\
\text { sèche } \\
\text { Dry matter }\end{array}$ & $\begin{array}{c}\text { Cendres } \\
A s h\end{array}$ & $\begin{array}{l}\text { Matières } \\
\text { azotées } \\
\text { Crude } \\
\text { protein }\end{array}$ & $\begin{array}{c}\text { Cellulose } \\
\text { brute } \\
\text { Crude fibre }\end{array}$ & $\begin{array}{l}\text { In vivo } \\
\text { (MS) } \\
\text { In vivo } \\
\text { (DM) }\end{array}$ & $\begin{array}{l}\text { In vitro I } 2 h \\
\text { In vitro I } 2 h\end{array}$ \\
\hline $57, \mathrm{I}$ & 39,6 & $\longrightarrow$ & 3,3 & I9,6 & 9,0 & I 4,5 & 26,8 & 64,2 & 49,0 \\
\hline $5^{0,5}$ & 49,0 & $\ldots$ & 0,5 & I 8, I & 8,8 & I 2,4 & 26,5 & $6 \mathrm{I}, \mathrm{o}$ & - \\
\hline 23,8 & 62,9 & 9,8 & 3,4 & 23,3 & 7,4 & 7,0 & 36,1 & 59,8 & 33,2 \\
\hline 37,0 & 60,0 & 1,0 & 2,0 & 20,3 & 7,6 & 9,7 & 35,6 & 57,2 & 33,5 \\
\hline 30,2 & $5^{8,7}$ & - & I I , I & I 9,2 & I 3,3 & I 6,6 & 23,5 & 59,4 & 42,6 \\
\hline 24,0 & $7 I, 5$ & - & 4,5 & I 7,2 & I 2,0 & I $2, \mathrm{I}$ & $24, I$ & $59, \mathrm{I}$ & $4^{0,4}$ \\
\hline 19,8 & 67,9 & 5,9 & 6,4 & 24,2 & 8,6 & 7,0 & 28,5 & 56,0 & 35,4 \\
\hline 28,0 & 62,5 & - & 9,5 & 20,3 & I 3,3 & 9,3 & 26,1 & 49,8 & 34,0 \\
\hline
\end{tabular}

ont été peu importantes sauf pour la teneur en matière sèche qui a été nettement plus faible au cours de la saison humide qu'en saison sèche $(-2,6$ points en moyenne).

Il est cependant intéressant de remarquer que, en liaison avec le pourcentage de feuilles, la teneur en matières azotées des fourrages était un peu plus faible pendant la saison humide que pendant la saison sèche, dans le cas des repousses jeunes (35 jours), alors qu'elle était un peu plus élevée dans le cas des repousses âgées $(56$ jours). La digestibilité de la matière sèche n'a pas présenté de liaison significative avec les paramètres de la composition chimique; en moyenne, elle a été plus faible au cours de la saison humide, puisqu'elle est passée de 59,8 p. Ioo en saison sèche à $56,8 \mathrm{p}$. Ioo en saison humide, mais cette différence a été très variable suivant la nature et l'âge du fourrage considéré; elle a été par exemple presque nulle pour le Digitaria à 35 jours (- 0,3 point), mais très importante pour la même plante âgée de 56 jours $(-6,2$ points). Ces variations, suivant la saison, de la digestibilité de la matière sèche ne peuvent pas être expliquées par celles des teneurs en matières azotées ou en cellulose brute. Seule la variation avec la saison de la teneur en cendres des repousses de Digitaria âgées de 56 jours explique peutêtre en partie la variation en sens inverse de la digestibilité de la matière sèche. Au niveau de la digestibilité in vitro I2 heures, le passage d'une saison à l'autre n'a entraîné que peu de variation : 37,6 en saison sèche contre 36,0 en saison humide (résultats correspondant à la moyenne de 3 fourrages). 


\section{Conditions climatiques}

La température a été plus élevée, de $\mathrm{I}^{\circ} \mathrm{C}$ en moyenne, au cours de la saison humide, l'augmentation la plus forte se situant entre I5 et Ig heures $\left(+2^{\circ} \mathrm{C}\right)$. Parallèlement, l'humidité relative, au cours de la saison humide, a été plus forte que pendant la saison sèche $(+4$ points) et cette variation a eu lieu principalement entre $\mathrm{I} 2$ et $\mathrm{I} 8$ heures.

L'indice de température effective, qui est un indicateur de stress, a été plus élevé de $\mathrm{I}, 3^{\circ} \mathrm{C}$ au cours de la saison humide, mais cette augmentation a été très variable suivant les périodes au cours desquelles ont été distribués les différents fourrages, respectivement 3,5 à $2,5^{\circ} \mathrm{C}$ avec le Brachiaria et le Digitaria à 35 jours, alors qu'elle était nulle ou même négative avec les deux autres fourrages.

\section{Quantités ingérées (tabl. 2)}

Les quantités ingérées, rapportées dans le tableau 2 , correspondent à la moyenne de deux semaines de mesure, les différences entre semaines n'étant pas significatives. Les fourrages ont cependant été ingérés en quantité très différente selon les animaux, le coefficient de variation étant en moyenne de 20 p. Ioo, quel que soit le fourrage considéré. Malgré cette variabilité importante entre animaux, les quantités ingérées sont significativement plus faibles au cours de la saison : $48,4 \mathrm{~g} / \mathrm{kg} \mathrm{P} \mathrm{P}^{0,75}$ contre 62,4 en saison sèche (différence significative à I p. Ioo, analyse de variance à 3 facteurs de variation). Cette influence de la saison a été variable suivant le fourrage considéré soit $-2 \mathrm{I}, 6 \mathrm{~g} / \mathrm{kg} \mathrm{P}^{0,75}$ pour le Brachiaria de 35 jours contre seulement $-7,2 \mathrm{~g} / \mathrm{kg} \mathrm{P}^{0,75}$ pour le Digitaria de 56 jours qui est pourtant le seul fourrage pour lequel on observe une chute non négligeable (-6.2 points) de la digestibilité en saison humide.

\section{Comportement alimentaire et mérycique (tab1. 2)}

Les durées journalières d'ingestion, comprises entre $3 \mathrm{I} 7$ et $403 \mathrm{mn}$, n'ont pas présenté de variations saisonnières significatives. Aussi, les variations de quantités de fourrage ingérées ont résulté en grande partie des variations de la durée unitaire d'ingestion ( $r=--0,944 ;$ tab1. 3). Cette dernière a été un peu plus élevée au cours de la saison humide : 7,37 $\mathrm{mn} / \mathrm{g} \cdot \mathrm{kg} \mathrm{P}^{0,75}$ que pendant la saison sèche : $6,02 \mathrm{mn} / \mathrm{g} . \mathrm{kg} \mathrm{P}^{0,75}$, mais la différence n'est pas significative.

Les durées d'ingestion des deux grands repas journaliers n'ont pas été identiques, celle du soir étant plus courte que celle du matin de $30 \mathrm{mn}$ environ, mais la variabilité entre animaux a été, en moyenne, élevée et plus forte au cours de la saison sèche que pendant la saison humide.

Le nombre de repas a varié de façon importante suivant la saison : en moyenne, les animaux ont fait $I, 7$ repas de moins en saison humide par rapport à la saison sèche $(\mathrm{P}<0,0 \mathrm{I})$, la différence atteignant 5,6 repas pour le Digitaria à 35 jours. La liaison entre le nombre de repas et les quantités ingérées par jour est élevée bien que non significative $(r=0,694)$.

La durée journalière de rumination a été en moyenne de $502 \pm 68 \mathrm{mn}$ et elle est indépendante de la durée journalière d'ingestion $(r=0,024)$; la durée de rumination n'a pas varié avec la saison, mais la variabilité entre animaux a été 


\begin{tabular}{|c|c|c|c|c|c|c|c|c|c|c|}
\hline \multirow{4}{*}{ 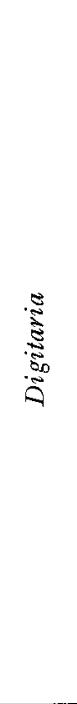 } & \multirow[b]{2}{*}{ in } & 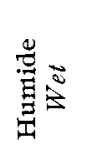 & 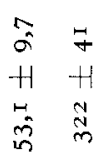 & 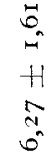 & $\begin{array}{l}\stackrel{D}{m} \\
+ \\
\Xi \\
\Xi\end{array}$ & 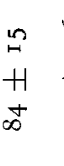 & 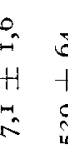 & & 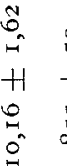 & \\
\hline & & 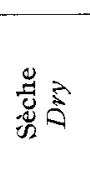 & 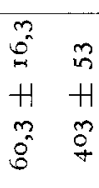 & $\begin{array}{l}0 \\
\stackrel{9}{-1} \\
-1 \\
\circ \\
8 \\
0\end{array}$ & 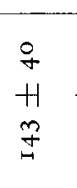 & $\begin{array}{l}\dot{0} \\
\dot{y} \\
+ \\
\vdots \\
\vdots \\
0\end{array}$ & $\begin{array}{l}y^{2} \\
i^{2} \\
y^{2} \\
x^{2}\end{array}$ & 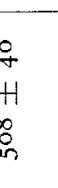 & 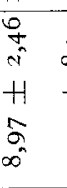 & \\
\hline & & 尊 & 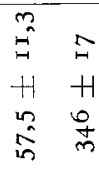 & 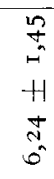 & 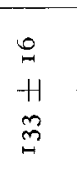 & 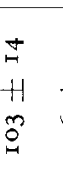 & $\begin{array}{l}0 \\
0 \\
\text { in } \\
\text { in }\end{array}$ & 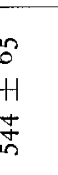 & 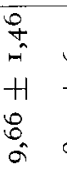 & \\
\hline & $\stackrel{m}{m}$ & 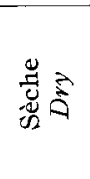 & 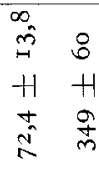 & $\begin{array}{l}b_{0} \\
0 \\
0 \\
+ \\
\infty \\
\infty \\
f\end{array}$ & $\begin{array}{l}\text { I } \\
+1 \\
+ \\
\infty\end{array}$ & $\begin{array}{l}\hat{m} \\
H \\
H \\
\Delta\end{array}$ & $\begin{array}{l}\hat{\theta} \\
\hat{i} \\
+ \\
\hat{H} \\
\hat{H}\end{array}$ & 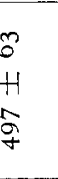 & $\begin{array}{l}b_{0}^{\circ} \\
0 \\
0 \\
+ \\
\vdots \\
0 \\
0\end{array}$ & \\
\hline \multirow{4}{*}{ 农 } & \multirow{2}{*}{ in } & 营 & 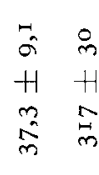 & $\begin{array}{l}\vec{b} \\
- \\
+ \\
\infty \\
\infty \\
\infty \\
\infty\end{array}$ & $\begin{array}{l}\hat{A} \\
H \\
\ddot{H}\end{array}$ & $\begin{array}{l}\infty \\
\stackrel{\infty}{\infty} \\
\infty \\
\infty\end{array}$ & $\begin{array}{l}\vec{G} \\
\vec{H} \\
\hat{\sigma}\end{array}$ & 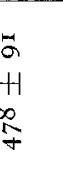 & 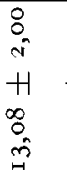 & 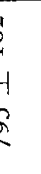 \\
\hline & & 咆 & 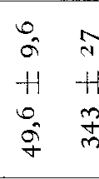 & 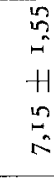 & $\begin{array}{l}\approx \\
+ \\
+ \\
心 \\
\end{array}$ & $\begin{array}{l}\stackrel{\circ}{\circ} \\
H \\
:\end{array}$ & $\begin{array}{c}2 \\
2 \\
\dot{m} \\
+ \\
0 \\
0\end{array}$ & $\begin{array}{l}\dot{q} \\
+ \\
+ \\
\sigma a \\
\sigma\end{array}$ & 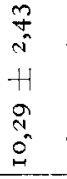 & $\begin{array}{l}b_{0} \\
t \\
0\end{array}$ \\
\hline & \multirow{2}{*}{$\stackrel{m}{m}$} & 茴 & 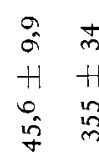 & 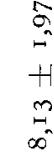 & $\begin{array}{l}0 \\
\text { H } \\
B \\
\Xi\end{array}$ & $\begin{array}{l}\text { o } \\
\text { H } \\
\text { gू }\end{array}$ & $\begin{array}{l} \pm \\
\vdots \\
+ \\
\vdots \\
\vdots\end{array}$ & $\begin{array}{l}8 \\
8 \\
+1 \\
b \\
0\end{array}$ & 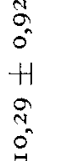 & t \\
\hline & & 窇高 & 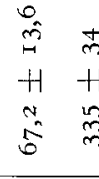 & 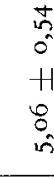 & $\begin{array}{l}+ \\
m \\
H \\
0 \\
0 \\
+\end{array}$ & $\begin{array}{l}\text { O } \\
\text { H } \\
\text { R }\end{array}$ & 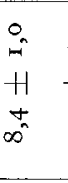 & $\begin{array}{l}5 \\
\text { in } \\
\text { on } \\
0 \\
\text { in }\end{array}$ & 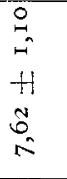 & \\
\hline 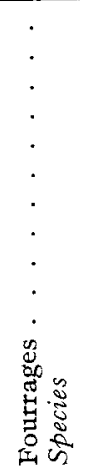 & 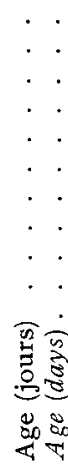 & 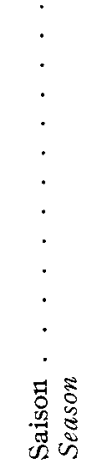 & 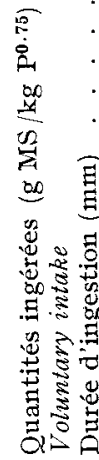 & : & है। & t & & & 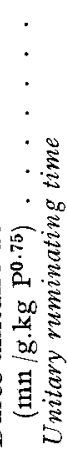 & \\
\hline
\end{tabular}




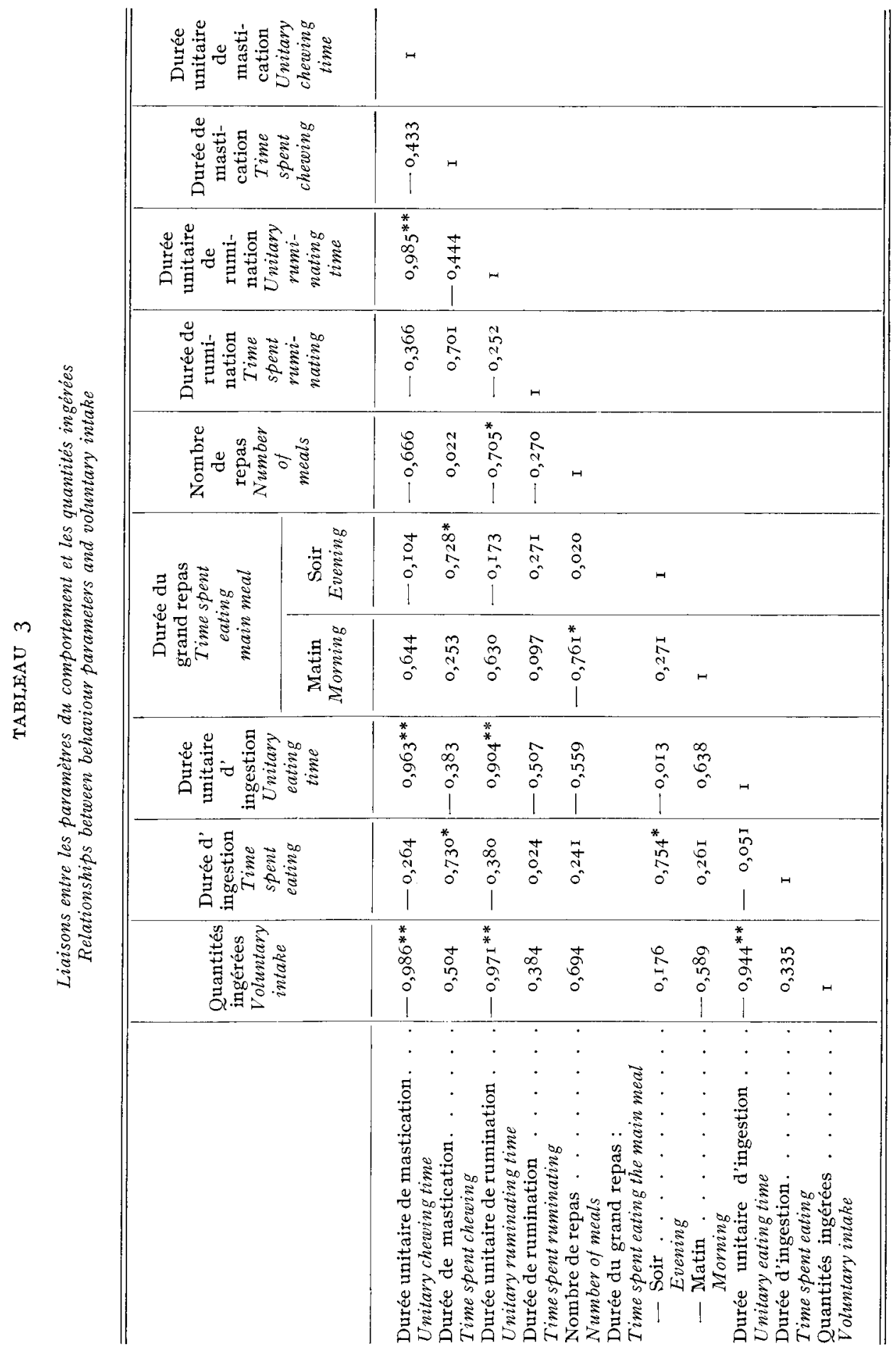


plus élevée au cours de la saison humide que pendant la saison sèche, ce qui est contraire à ce qu'on a trouvé pour les autres paramètres du comportement. La durée unitaire de rumination, comme la durée unitaire d'ingestion, a été plus élevée au cours de la saison humide : Io,80 mn $/ g . k g \mathrm{P}^{0,75}$ que pendant la saison sèche: $8,46 \mathrm{mn} / \mathrm{g} . \mathrm{kg} \mathrm{P} \mathrm{P}^{0,75}$ (différence significative, $\mathrm{P}<\mathrm{o,or}$ ).

La durée journalière de mastication (ingestion + rumination) a varié de 795 à gI I mn, et comme les durées d'ingestion et de rumination, elle a été indépendante de la saison, alors que la durée unitaire de mastication a varié de façon significative avec la saison : $\mathrm{I} 4,44 \mathrm{mn} / \mathrm{g} . \mathrm{kg} \mathrm{P}^{0,75}$ en saison sèche contre $\mathrm{I} 8$, I 9 en saison humide, et était en liaison étroite avec les quantités ingérées $\left(r=0,986^{* *}\right.$, différence significative, $\mathrm{P}<\mathrm{O}, \mathrm{OI})$.

\section{Discussion}

Il est difficile de comparer les résultats de cette étude à ceux obtenus en zone tempérée, les espèces végétales et les conditions climatiques étant différentes. Cependant, on peut constater que les quantités de fourrage ingérées dans ces 4 essais $\left(55,4 \mathrm{~g} / \mathrm{kg} \mathrm{P}^{0,75}\right.$ en moyenne) sont peu différentes des quantités de fourrage vert de graminées, de digestibilité comparable, ingérées par des moutons en zone tempérée $\left(55 \mathrm{~g} / \mathrm{kg} \mathrm{P}^{0.75}\right.$, Demarquiliy, JARRIGE, I97I). Mais les quantités de fourrage ingérées présentent des variations saisonnières importantes : elles sont plus faibles en moyenne de $22 \mathrm{p}$. Ioo au cours de la saison humide, alors que JoHNSON et al., (r968) ne trouvent aucune influence de la saison sur les quantités de fourrage ingérées par des bovins aux Philippines. Cependant, Butternorth, Groom et WILSON (I96I), à Trinidad, mettent comme nous en évidence une diminution de l'ingestion au cours de la saison humide avec des bovins pâturant des repousses de Pangola.

Quelles peuvent être les causes de ces variations saisonnières, la saison agit-elle directement sur l'appétit des animaux ou modifie-t-elle l'ingestibilité du fourrage?

On sait d'après les études réalisées en zone tempérée que l'ingestibilité d'une espèce fourragère donnée dépend avant tout de la digestibilité (BLAXTER, WAINMAN et WILSON, I96I; DEMARQUIILY, I965) et donc de la composition chimique (teneurs en matières azotées et cellulose brute) de la plante. Mais, dans ces essais, les variations saisonnières de digestibilité sont faibles et d'ailleurs indépendantes des variations de composition chimique de la plante qui restent elles-mêmes très limitées, notamment en ce qui concerne la cellulose brute.

D'autre part BRODY (I956) a montré que les variations de température et d'humidité auxquelles sont soumis les animaux au cours de la saison humide peuvent entraîner une modification de leur capacité d'ingestion. En effet, de nombreux travaux réalisés à la Station du Missouri (RAGSDALE et al., I950; WAYMAN et al., I g62) ont permis de mettre en évidence qu'une augmentation de température entraînait une diminution de consommation. Mais tous les essais ont été menés en chambre conditionnée, c'est-à-dire avec des animaux maintenus sous un stress d'intensité constante; or, PAYNE (I966) suggère 1'existence, sous climat tropical, d'un mécanisme de compensation la nuit, la température étant un peu plus faible que le jour, ce qui limite 1'effet du stress thermique.

Dans ce travail on observe, lors des essais réalisés avec des fourrages âgés 
de 35 jours, une variation de 1'indice de température effective (fig. 2) qui pourrait expliquer en partie la diminution de consommation du fourrage au cours de la saison humide; mais les variations de la teneur en matières azotées et (ou) de la digestibilité peuvent être aussi des facteurs explicatifs partiels de ces variations de consommation. Pour les fourrages âgés de 56 jours, ni les modifications de la teneur en matières azotées, ni celles de la température effective ne peuvent être à l'origine de la diminution des quantités de fourrage ingérées. Seules les variations de digestibilité peuvent expliquer la baisse d'ingestibilité, notamment pour le Digitaria, mais seulement très partiellement pour le Brachiaria âgé de 56 jours (fig. 2). Il est à noter cependant que les variations de température effective ne

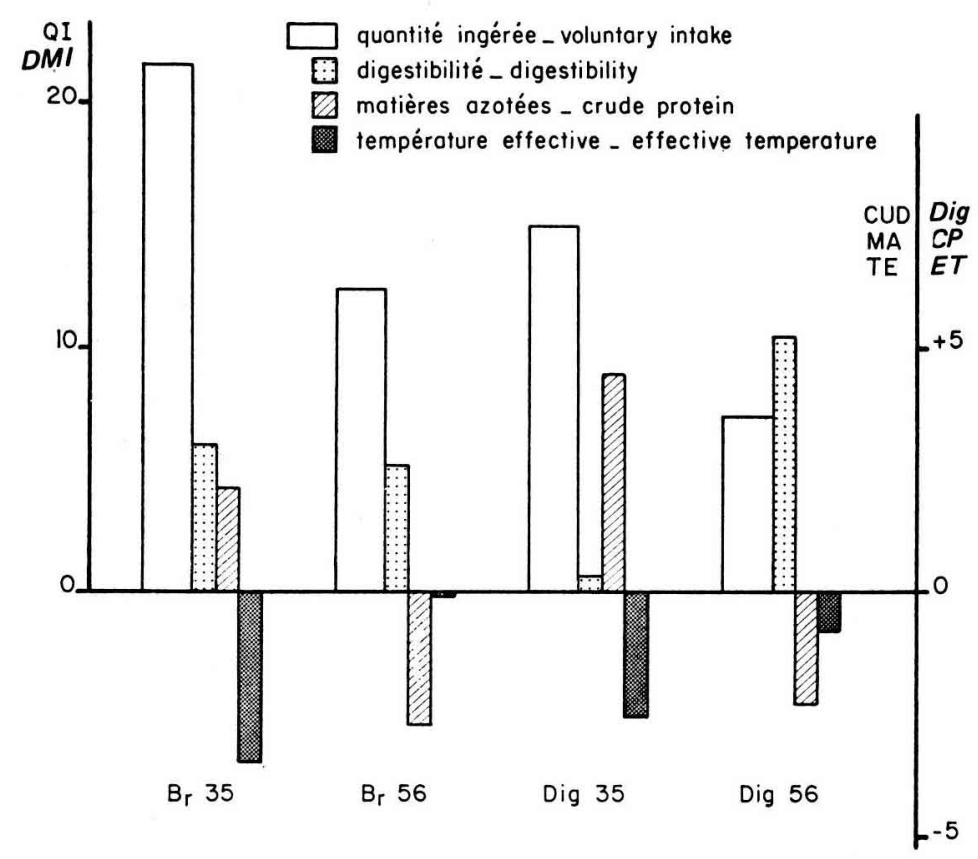

FIG. 2. - Représentation graphique des variations saisonnières (saison sèche - saison humide) des quantités ingérées, de digestibilité, de teneurs en matières azotées et de l'indice de température effective.

Graph of seasonal variations (dry season - wet season) in voluntary intake, digestibility, crude protein and effective temperature.

représentent pas au mieux l'état de confort thermique de 1'animal; d'une part il s'agit d'une valeur moyenne, or les variations nycthémérales sont importantes; d'autre part cet indice permet de regrouper les différents facteurs climatiques mais il ne fait pas intervenir les caractéristiques physiologiques ou zootechniques de l'animal comme le "facteur de réduction de gain " ou GRF de MORRIson par exemple (BERBIGIER, I978). Même si 1'indice de température effective ne varie pas, il n'est donc pas possible pour autant d'affirmer que les variations saisonnières de quantités ingérées ne dépendent pas d'une modification de la capacité d'ingestion des moutons. Enfin l'influence de la saison peut s'expliquer aussi par l'intermé- 
diaire des variations de la teneur en eau des fourrages ou de leur appétibilité (qualités organoleptiques) qu'il est difficile d'apprécier.

En définitive, pour confirmer une action directe du climat sur l'appétit des animaux, il faudrait pouvoir distribuer le même fourrage (un foin, par exemple) au cours des deux saisons sèche et humide. Il serait intéressant d'autre part d'étudier la distribution nycthémérale de l'ingestion et de la mettre en parallèle avec les variations journalières de l'indice de température effective, ce qui n'a pu être fait dans cette étude puisque les repas n'ont pas toujours été distribués à heure fixe.

\title{
Accepté pour publication en octobre 1979
}

\author{
Summary \\ Influence of the season on the feeding behaviour \\ of sheep receiving green forage in a wet tropical zone.
}

Seasonal variations in dry matter intake as well as changes in the eating and ruminating behaviour of sheep fed regrowths of 35 or 56 day-old Brachiaria decumbens and Digitavia swazilandensis were studied during the two dry and wet seasons.

The voluntary intake was significantly lower during the wet season: $48.4 \mathrm{~g} / \mathrm{kg}^{0,75}$ than during the dry season: $62.4 \mathrm{~g} / \mathrm{kg} \mathrm{W}^{0.75}$. The dry matter digestibility varied from $59.8 \mathrm{p}$. 100 in the wet season to $56.8 \mathrm{p}$. I 100 in the dry season. The crude protein content of 35 -day regrowths was lower during the wet season: 12.2 p. Ioo versus I 5.5 in the dry season. On the other hand, the "effective temperature index" of the thermal comfort index calculated in terms of temperature and humidity was higher, on an average, during the wet season $\left(+\mathrm{I} \cdot 3^{\circ} \mathrm{C}\right)$. The increase

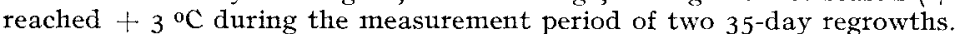

The seasonal variations of the unitary eating, ruminating and chewing time ranged from $7.37 \mathrm{mn} / \mathrm{g} . \mathrm{Kg} \mathrm{W}^{0.75}$ in the wet season to 6.02 in the dry season from Io.80 to 8.46 and I8. I9 to I 4.44 respectively and the number of meals from 6.7 to 8.4

The seasonal variations in the level of feed intake are difficult to explain. They seem to depend both on changes in the climatic conditions (variations in the effective temperature index, although this criterion might not be characteristic of the optimum thermal comfort of the animal) leading to a modification of the voluntary feed intake of the animals and on variations in the digestibility and crude protein content of the forage, i.e. its level of voluntary intake.

\section{Références bibliographiques}

BERBIGIER P., 1978. Échanges thermiques des animaux d'élevage en fonction des paramètres climatiques. Bull. Tech. Inf., 328-329, I 29 -I 5 I.

Blaxter K. L., Wainman F. W., WiLson R. S., I96I. The regulation of food intake by sheep. Anim. Prod., 13, 5I-6r.

BRODY S., I956. Climatic physiology of cattle. J. Dairy Sci., 39, 7 I 5-725.

Butterworth M. H., Groom C. G., Wirson P. N., Ig6I. The intake of Pangola grass (Digitaria decumbens Stent.) under wet and dry season conditions in Trinidad. J. Agric. Sci., 56, 407-4ro.

Chenost M., I972. Observations préliminaires sur les variations saisonnières de la quantité d'aliment ingérée par les caprins en milieu tropical humide. Ann. Zootech., 21, I I 3-120.

DEMARQUILLY C., I 965 . Factors affecting the voluntary intake of green forage by sheep. Proc. gth Intern. Grassld. Congr., Sao Paulo, 877-885.

DEMARQUILLY C., JARRIGE R., I97I. The digestibility and intake of forages from artificial and natural grassland. Gen. Meeting of European Grassld. Assoc., Lausanne, 9I-Io6. 
Johnson W. L., Hardison W. A., Ordoveza A. L., Castilifo L. S., i968. The nutritive value of Panicum maximum (Guinea grass). III. - Factors affecting voluntary intake by cattle of water buffaloes. J. Agric. Sci., 71, 67-71.

MIL,FORD R., Minson D. J., 1965. Intake of tropical pasture species. Proc. gth Intern. Grassid. Congr., Sao Paulo, 81 5-822.

MINSON D. J., MILIORD R., I967. In vitro and faecal nitrogen techniques for predicting the voluntary intake of Chloris gayana. J. Brit. Grassld Soc., 22, I 70-I 75.

PaYNe W. J. A., I966. Nutrition of ruminants in the tropics. Nutr. Abstr. Rev., 36, 653-67o.

Ragsdal, A. C., Thompson H. J., Worstel. D. M., Brody S., 1950. Environmental physiology with special reference to domestic animals. IX. - Milk production and feed and water consumption responses of Brahman, Jersey and Holstein cows to change in temperature $50^{\circ}$ to $105^{\circ} \mathrm{F}$ and $50^{\circ}$ to $8^{\circ} \mathrm{F}$. Missouri A gr. Exp. Stat. Research Bull., 460, I-27.

RUCKEBUSCH Y., I963. Recherches sur la régulation centrale du comportement alimentaire chez les ruminants. Thèse Doct. Sci., Univ. Lyon.

TILLEY J. M. A., TERRY R. A., I963. A two-stage technique for in vitro digestion of forage crops. J. Brit. Grassld Soc., 18, IO4-I I I.

Wayman O., Johnson H. D., Merilan C. P., Berrky J. L., AERd, I962. Effects of ad libitum or force-feeding of two rations on lactating dairy cows subject to temperature stress. J. Dairy Sci., 45, I $472-\mathrm{I} 478$. 